\title{
Research Article New CZM for Interfacial Crack Growth
}

\author{
Huifen Peng, Yujie Song, and Ye Xia \\ College of Mechanical Science and Engineering, Northeast Petroleum University, Daqing 163318, China \\ Correspondence should be addressed to Yujie Song; syjdqpi@163.com
}

Received 7 April 2016; Revised 4 July 2016; Accepted 12 December 2016; Published 18 January 2017

Academic Editor: Franck J. Vernerey

Copyright (C) 2017 Huifen Peng et al. This is an open access article distributed under the Creative Commons Attribution License, which permits unrestricted use, distribution, and reproduction in any medium, provided the original work is properly cited.

The cohesive zone model (CZM) has been widely used for numerical simulations of interface crack growth. However, geometrical and material discontinuities decrease the accuracy and efficiency of the CZM when based on the conventional finite element method (CFEM). In order to promote the development of numerical simulation of interfacial crack growth, a new CZM, based on the wavelet finite element method (WFEM), is presented. Some fundamental issues regarding CZM of interface crack growth of double cantilever beam (DCB) testing were studied. The simulation results were compared with the experimental and simulation results of CFEM. It was found that the new CZM had higher accuracy and efficiency in the simulation of interface crack growth. At last, the impact of crack initiation length and elastic constants of material on interface crack growth was studied based on the new CZM. These results provided a basis for reasonable structure design of composite material in engineering.

\section{Introduction}

Interface in the composite materials is the common surface area of each connecting phase and it contributes to the transmission of mechanical property. Numerous studies have shown that failure often occurs along the interface; therefore, the fracture behavior of interface crack growth attracted considerable attention in recent years [1-4]. Numerical simulation is the main method for interface fracture analysis, and it is also the key to the development of interface fracture mechanics. At present, many scholars, both within the country and abroad, have carried out studies on the problem of interface crack growth, by using different numerical methods [5-9]. Although many numerical calculation methods for interface crack growth have already been reported, as indicated above, the number of papers that simulates interface crack growth based on WFEM is limited.

In most of the finite element applications of the CZM, it is natural that WFEM is a new numerical calculation method which has been gaining lot of interest in the last decade. It uses the scaling function or the wavelet function as interpolation function instead of the traditional polynomial, and its main characteristics are as follows: lower undetermined coefficient, higher approximation accuracy, strong localization performance, and multiresolution analysis. Regarding the problem of crack growth, in terms of not changing mesh dividing, the accuracy and efficiency of numerical calculation can be improved by increasing the node information, and it has better adaptive performance. Therefore, the method has an application prospect in the aspect of numerical simulation of crack growth. Zuo et al. [10] study static and free vibration problems of laminated composite plates adopting WFEM and higher-order plate theory, the effects of lengthto-thickness ratios, layer numbers, and fiber orientations on the deflections and frequencies; the results showed that WFEM provides better results and that it is accurate and stable for free vibration analysis of laminated composite plates. Xiang et al. [11] constructed plane plate element using Hermite cubic spline wavelet for stress intensity factors (SIFs) evaluation in cracked plate structures; numerical simulation showed WFEM has better localization property. Chen et al. [12] proposed a second generation WFEM for diagnosing rotors with different crack location and size; the experimental results denote that the proposed method has higher identification precision. Chen et al. [13] proposed a novel numerical algorithm of crack fault prognosis based on WFEM and verified the effectiveness and accuracy of the proposed method through experiments. In short, WFEM has been successfully applied to various fields of engineering numerical analysis [14-19]. 
CZM is an effective approach for simulating fracture events. In recent years, CZM was studied and some excellent achievements have been obtained [20-23].

According to the current known researching achievement, the combination of WFEM with CZM for simulating interface crack growth has not been studied. In this paper, by combining the WFEM and cohesive model, the stiffness matrix of wavelet cohesive interface element was derived. The strain energy release rate (SERR) was calculated by using the virtual crack closure technique (VCCT) $[24,25]$, the process of crack growth was described by the nonlinear fracture criterion [26], and the experimental results and simulation results of CFEM were compared. Finally, the impact of initial length of interface crack and elastic modulus of material on interface crack growth was studied.

\section{B-Spline Wavelet on the Interval $[0,1]$}

In order to avoid the numerical oscillation phenomenon of classical wavelet when solving boundary value problem, American scholars Chui and Quak [27] presented the Bspline wavelet on the interval (BSWI). The 0 scale $m$ th-order BSWI functions and wavelets were given by Goswami et al. [28]. For the needs of the following part, in this paper, the 0 scale 4 th-order scaling functions and wavelets were given in the following:

$$
\begin{aligned}
& \phi_{4,-3}^{0}(x)=\frac{1}{6} \begin{cases}6-18 x+18 x^{2}-6 x^{3}, & x \in[0,1] \\
0, & \text { the rest, }\end{cases} \\
& \phi_{4,-2}^{0}(x)=\frac{1}{6} \begin{cases}18 x-27 x^{2}+\frac{21}{2} x^{3}, & x \in[0,1] \\
12-18 x+9 x^{2}-\frac{3}{2} x^{3}, & x \in[1,2] \\
0, & \text { the rest, }\end{cases} \\
& \phi_{4,-1}^{0}(x)=\frac{1}{6} \begin{cases}9 x^{2}-\frac{11}{2} x^{3}, & x \in[0,1] \\
-9+27 x-18 x^{2}+\frac{7}{2} x^{3}, & x \in[1,2] \\
27-27 x+9 x^{2}-x^{3}, & x \in[2,3] \\
0, & \text { the rest, }\end{cases} \\
& \phi_{4,0}^{0}(x)=\frac{1}{6} \begin{cases}x^{3}, & x \in[0,1] \\
4-12 x+12 x^{2}-3 x^{3}, & x \in[1,2] \\
-44+60 x-24 x^{2}+3 x^{3}, & x \in[2,3] \\
64-48 x+12 x^{2}-x^{3}, & x \in[3,4] \\
0, & \text { the rest. }\end{cases}
\end{aligned}
$$

The 0 scale 4 th-order wavelet functions can be obtained by the following function:

$$
5040 \times \psi_{4, k}^{0}(x)=\sum_{i=0}^{3} a_{i} x^{j},
$$

where $a_{i}$ is the corresponding coefficient to different $k(-3,-2,-1,0)$, and it can be obtained by LUT [29].
In order to have at least one inner wavelet, the following condition must be satisfied:

$$
2^{j} \geq 2 m-1
$$

where $m$ and $j$ are the order and scale of BSWI, respectively.

Since the 0 scale $m$ th-order scaling and wavelet functions have been obtained, the corresponding $j$ scale $m$ thorder of BSWI $\left(\mathrm{BSWI}_{m j}\right)$ scaling functions $\phi_{m, k}^{j}(\xi)$ and the corresponding wavelet functions $\psi_{m, k}^{j}(\xi)$ can be evaluated by the following equations:

$$
\begin{aligned}
& \phi_{m, k}^{j}(\varepsilon) \\
& = \begin{cases}\phi_{m, k}\left(2^{j} \xi\right), & k=-m+1, \ldots,-1 \\
\phi_{m, 2^{j}-m-k}\left(1-2^{j} \xi\right), & k=2^{j}-m+1, \ldots, 2^{j}-1 \\
\phi_{m, 0}\left(2^{j} \xi-k\right), & k=0, \ldots, 2^{j}-m,\end{cases} \\
& \psi_{m, k}^{j}(\xi) \\
& = \begin{cases}\psi_{m, k}\left(2^{j} \xi\right), & k=-m+1, \ldots,-1 \\
\psi_{m, 2^{j}-2 m-k+1}\left(1-2^{j} \xi\right), & k=2^{j}-2 m+2, \ldots, 2^{j}-m \\
\psi_{m, 0}\left(2^{j} \xi-k\right), & k=0, \ldots, 2^{j}-2 m+1,\end{cases}
\end{aligned}
$$

where $\xi$ is the independent variable of scaling and wavelet functions on the interval $[0,1]$. Therefore, the scaling functions can be obtained in vector form as follows:

$$
\boldsymbol{\Phi}=\left[\begin{array}{lllll}
\phi_{m,-m+1}^{j} & (\xi) & \phi_{m,-m+2}^{j}(\xi) & \cdots & \phi_{m, 2^{j}-1}^{j}(\xi)
\end{array}\right]
$$

where $\phi_{m,-m+1}^{j}(\xi), \phi_{m,-m+2}^{j}(\xi), \ldots, \phi_{m, 2^{j}-1}^{j}(\xi)$ are the scaling functions obtained from (4). The wavelet functions can also be obtained in vector form:

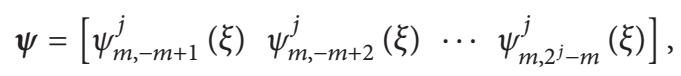

where $\psi_{m,-m+1}^{j}(\xi), \psi_{m,-m+2}^{j}(\xi), \ldots, \psi_{m, 2^{j}-m}^{j}(\xi)$ are the wavelet functions obtained from (5).

In order to construct the two-dimensional wavelet plane plate element, the two-dimensional BSWI scaling function is required. Through the tensor product, the two-dimensional scaling functions are

$$
\Phi=\Phi_{1} \otimes \Phi_{2}
$$

where $\boldsymbol{\Phi}_{1}=\left[\phi_{m,-m+1}^{j}(\xi) \phi_{m,-m+2}^{j}(\xi) \cdots \phi_{m, 2^{j}-1}^{j}(\xi)\right]$ is the one row vector combined with the scaling functions for $m$ at the scale $j$ and $\boldsymbol{\Phi}_{2}=\left[\begin{array}{llll}\phi_{m,-m+1}^{j}(\eta) & \phi_{m,-m+2}^{j}(\eta) & \cdots & \phi_{m, 2^{j}-1}^{j}(\eta)\end{array}\right]$ is another row vector combined with the $j$ scale $m$ th-order scaling functions. $\otimes$ is the Kronecker symbol.

The two-dimensional wavelet functions are

$$
\begin{aligned}
& \Psi^{1}=\Phi_{1} \otimes \Psi_{2}, \\
& \Psi^{2}=\Phi_{2} \otimes \Psi_{1}, \\
& \Psi^{3}=\Psi_{1} \otimes \Psi_{2},
\end{aligned}
$$

where $\Phi_{1}$ and $\Phi_{2}$ are the vector forms of scaling functions and $\Psi_{1}$ and $\Psi_{2}$ are wavelet functions. 


\section{Wavelet Cohesive Zone Model}

The CZM is a simplified model of the interface layer. The interface characteristics are described by the relationship between the interface traction and the relative displacement of upper and lower interface. The CZM is based on elasticplastic fracture mechanics and is an effective approach for simulating fractures, which can potentially avoid the stress singularity at the crack tip in linear elastic fracture mechanics, and the crack interface stress and fracture energy can be therefore obtained. As the research and development of CZM evolves, two main CZMs have been proposed and have already been used for the simulating of ductile and two-phase material interface crack growth [30-32].

3.1. The Bilinear CZM. The relationship between normal cohesive traction $T_{n}$ and normal displacement jump $\delta_{n}$ can be expressed as

$$
T_{n}=K_{n} \delta_{n}\left(1-D_{n}\right) .
$$

The normal critical fracture energy values are computed as

$$
\varphi_{n}^{c}=\frac{1}{2} T_{n}^{\max } \delta_{n}^{c},
$$

where $K_{n}$ is the normal cohesive stiffness $T_{n}^{\max } / \delta_{n}^{*}, T_{n}^{\max }$ is the maximum normal cohesive traction, $\delta_{n}^{*}$ is the normal displacement jump at the maximum normal cohesive traction, and $\delta_{n}^{c}$ is the normal displacement jump at the completion of debonding. $D_{n}$ is the interface damage parameter and is an irreversible quantity. The value of $D_{n}$ ranges from 0 to 1 ; when $D_{n}=1$, the interface is completely out of bond and is defined as

$$
D_{n}= \begin{cases}0, & \delta_{n} \leq \delta_{n}^{*} \\ \left(\frac{\delta_{n}-\delta_{n}^{*}}{\delta_{n}}\right)\left(\frac{\delta_{n}^{c}}{\delta_{n}^{c}-\delta_{n}^{*}}\right), & \delta_{n}^{*}<\delta_{n} \leq \delta_{n}^{c} \\ 1, & \delta_{n} \geq \delta_{n}^{c},\end{cases}
$$

where $\delta_{n}^{c}$ is the normal displacement jump at the completion of debonding.

Figure 1 shows the relationship between interface normal cohesive traction $T_{n}$ and normal displacement jump $\delta_{n}$. It clearly shows that, with the increase of normal displacement jump $\delta_{n}$, when the interface layer is detached, the interface cohesive traction is increased, reaching a maximum value and then decreasing to zero in the end.

When the normal displacement $\delta_{n} \geq \delta_{n}^{c}$, then $T_{n}=0$. The interface has been completely detached and has lost the ability of load transferring; thus, the damage process of interface layer can be simulated by this curve.

3.2. Wavelet CZM Interface Element. With the aim to converge all difficulties in CZM numerical analyses, a method of combining of WFEM with CZM has been proposed, where wavelet CZM interface element is used to simulate the interface separation process. In this paper, as an example, twodimensional wavelet interface elements were used to deduce

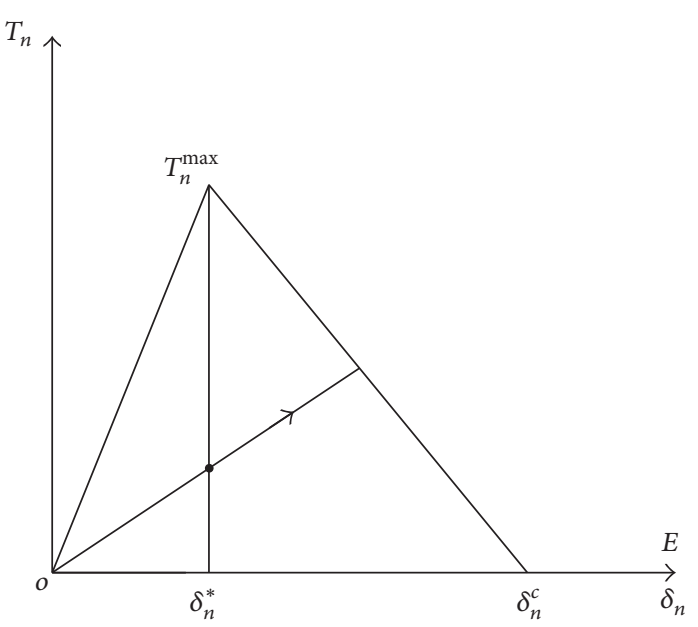

FIGURE 1: The bilinear CZM.

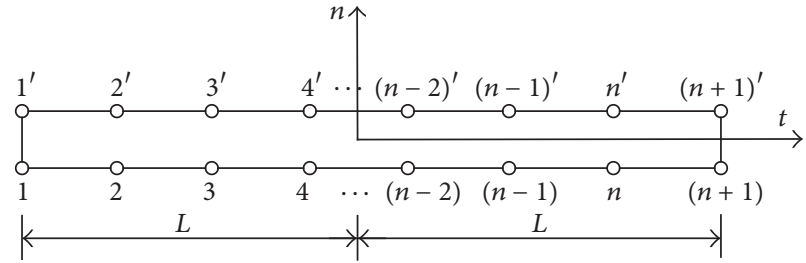

FIGURE 2: Wavelet interface elements.

the wavelet interface element stiffness matrix. By considering the $j$ scale $m$ th order of the BSWI scaling function as the interpolation function, an interface element was constructed, and its structure is presented in Figure 2. The element contains $2(n+1)$ nodes, and each node has two degrees of freedom, which includes horizontal and vertical direction. In applications, the upper nodes of interface initially coincide with the lower nodes, which mean that the initial thickness of interface element is 0 .

The definition of the wavelet interface element nodes displacement in the standard solution domain is as follows:

$$
\mathbf{u}^{e}=\left[u_{t}^{1}, u_{n}^{1}, u_{t}^{2}, u_{n}^{2}, \ldots, u_{t}^{(n+1)}, u_{n}^{2(n+1)}\right]^{T} .
$$

The continuous displacement field of upper and lower interface of wavelet interface element is

$$
u=\left[u_{t}^{-}, u_{n}^{-}, u_{t}^{+}, u_{n}^{+}\right]^{T},
$$

where "+", “-“ represent the upper and lower displacement of wavelet interface element, respectively.

When using the interval B-spline wavelet scaling function of the $j$ scale $m$ th order as an interpolation function, the displacement interpolation function is

$$
u(\xi)=\sum_{k=-m+1}^{2^{j}-1} a_{m, k}^{j} \phi_{m, k}^{j}(\xi)=\Phi \mathbf{a}^{e},
$$

where $\xi$ is the tangential coordinates along with the wavelet interface element in the standard solution domain, the 
wavelet coefficient $\mathbf{a}^{e}=\left[\begin{array}{llll}a_{m,-m+1}^{j} & a_{m,-m+2}^{j} & \cdots & a_{m, 2^{j}-1}^{j}\end{array}\right]$, and the wavelets scaling function can be obtained in vector form

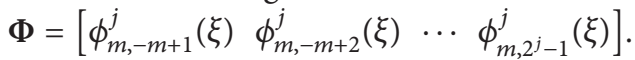

After matrix conversion, the displacement interpolation function represented by the nodal displacement array is

$$
u(\xi)=\Phi\left(\mathbf{R}^{e}\right)^{-1} \mathbf{u}^{e}=\mathbf{N}^{e} \mathbf{u}^{e},
$$

where the converted matrix $\mathbf{R}^{e}=$ $\left[\begin{array}{llll}\boldsymbol{\Phi}^{T}\left(\xi_{1}\right) & \boldsymbol{\Phi}^{T}\left(\xi_{2}\right) & \cdots & \boldsymbol{\Phi}^{T}\left(\xi_{n+1}\right)\end{array}\right]^{T}$. The normal displacement field is

$$
\delta=\left(\delta_{t}, \delta_{n}\right)^{T}=\left[u_{t}^{+}-u_{t}^{-}, u_{n}^{+}-u_{n}^{-}\right]^{T}=\mathbf{L} \cdot \mathbf{N}^{e} \cdot \mathbf{u}_{e},
$$

where

$$
\begin{aligned}
\mathbf{L} & =\left[\begin{array}{cccc}
-1 & 1 & 0 & 0 \\
0 & 0 & -1 & 1
\end{array}\right] ; \\
\mathbf{N}^{e} & \\
& =\left[\begin{array}{cccccccccccccccccccccc}
N_{1}^{e} & 0 & N_{2}^{e} & 0 & \cdots & N_{n-1}^{e} & 0 & N_{n}^{e} & 0 & N_{n+1}^{e} & 0 & 0 & 0 & 0 & 0 & 0 & 0 & \cdots & 0 & 0 & 0 & 0 \\
0 & N_{1}^{e} & 0 & N_{2}^{e} & \cdots & 0 & N_{n-1}^{e} & 0 & N_{n}^{e} & 0 & N_{n+1}^{e} & 0 & 0 & 0 & 0 & 0 & 0 & \cdots & 0 & 0 & 0 & 0 \\
0 & 0 & 0 & 0 & \cdots & 0 & 0 & 0 & 0 & 0 & 0 & N_{n+1}^{e} & 0 & N_{n}^{e} & 0 & N_{n-1}^{e} & 0 & \cdots & N_{2}^{e} & 0 & N_{1}^{e} & 0 \\
0 & 0 & 0 & 0 & \cdots & 0 & 0 & 0 & 0 & 0 & 0 & 0 & N_{n+1}^{e} & 0 & N_{n}^{e} & 0 & N_{n-1}^{e} & \cdots & 0 & N_{2}^{e} & 0 & N_{1}^{e}
\end{array}\right] .
\end{aligned}
$$

The wavelet interface element stiffness matrix is

$$
\mathbf{K}=\int_{-L}^{L} \mathbf{M} \cdot \mathbf{S} \cdot \mathbf{L} \cdot \mathbf{N}^{e} d \xi
$$

where

$$
\mathbf{M}=\left[\begin{array}{cccccccccccccccccccc}
N_{1}^{e} & 0 & N_{2}^{e} & 0 & \cdots & N_{n-1}^{e} & 0 & N_{n}^{e} & 0 & N_{n+1}^{e} & 0 & N_{n}^{e} & 0 & N_{n-1}^{e} & 0 & \cdots & N_{2}^{e} & 0 & N_{1}^{e} & 0 \\
0 & N_{1}^{e} & 0 & N_{2}^{e} & \cdots & 0 & N_{n-1}^{e} & 0 & N_{n}^{e} & 0 & -N_{n+1}^{e} & 0 & -N_{n}^{e} & 0 & -N_{n-1}^{e} & \cdots & 0 & -N_{2}^{e} & 0 & -N_{1}^{e}
\end{array}\right]^{T} ;
$$

additionally

$$
\mathbf{S}=\left[\begin{array}{ll}
\frac{\partial T_{t}}{\partial \delta_{t}} & \frac{\partial T_{t}}{\partial \delta_{n}} \\
\frac{\partial T_{n}}{\partial \delta_{t}} & \frac{\partial T_{n}}{\partial \delta_{n}}
\end{array}\right]
$$

is the interface elasticity matrix; $T_{t}$ and $T_{n}$ are the tangential and normal cohesive traction, respectively; $\delta_{t}$ and $\delta_{n}$ are the tangential and normal displacement jump, respectively.

\section{Numerical Examples}

4.1. Interface Crack Growth Problem of DCB Testing. DCB model is made of a steel plate of length $L=150 \mathrm{~mm}$ and width $H=4 \mathrm{~mm}$ and crack length $a=15 \mathrm{~mm}$ at the free end of DCB; the SERRs are $G_{\text {IC }}=600 \mathrm{~J} / \mathrm{m}^{2}, G_{\text {IIC }}=800 \mathrm{~J} / \mathrm{m}^{2}$, and $G_{\text {IIIC }}=800 \mathrm{~J} / \mathrm{m}^{2}$, and the properties of both DCB materials and interfaces are presented in Table 1.

By taking advantage of two $\mathrm{BSWI}_{43}$ wavelet plane plate elements to model the upper and lower solid beam, respectively, and one wavelet cohesive interface element to model the interface for the DCB, a wavelet finite element model of crack growth was established, as demonstrated in Figure 3.
The process of interface crack growth was simulated by the nonlinear fracture criterion.

When material 1 and material 2 are the same material, Table 2 shows the relative error of the WFEM calculation results compared to the CFEM calculation results, and it indicated that the WFEM can obtain higher accuracy with less elements and nodes and that it is suitable to solve the problem of interface crack growth.

Figure 4 presents the changing trends of the interface reaction forces as a function of time, for different elastic moduli. As presented in Figure 4, for different elastic moduli, the interface reaction forces experienced a hardening process of gradually reaching their maximum value. The interface cracks were propagated, during the hardening process, which required the increase of external force of the interface, which, subsequently, resulted into crack growth.

In order to study the impact of elastic modulus ratio on interface crack growth, it is supposed that the interface strength is lower than that of material 1 and material 2. The interface crack was extended along the predefined interface, and the elastic modulus of material 1 was $135 \mathrm{MPa}$, while the elastic modulus of material 2 was altered, without changing other parameters. Regarding the wavelet interface element, the variation rule of stress at the crack tip was obtained in 


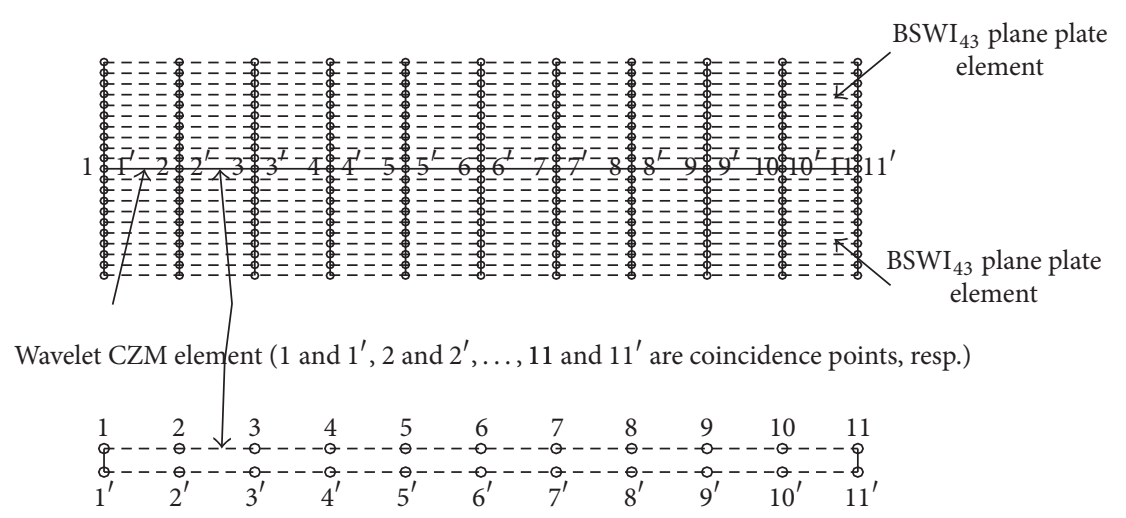

Figure 3: Schematic for elements and nodes of DCB.

TABLE 1: Properties of material 1 and interface.

\begin{tabular}{lc}
\hline Material 1 & Interface \\
\hline$E_{1}=120 \mathrm{GPa}, E_{2}=E_{3}=10.5 \mathrm{GPa}$ & $\sigma_{\max }=2.4 \times 10^{5} \mathrm{~Pa}, \tau_{\max }=4.2 \times 10^{5} \mathrm{~Pa}$ \\
$v_{12}=v_{13}=0.3, v_{23}=0.51$ & $K_{n}=1 \times 10^{5} \mathrm{~N} / \mathrm{m}, K_{t}=1 \times 10^{5} \mathrm{~N} / \mathrm{m}$ \\
$G_{12}=G_{13}=5.25 \mathrm{GPa}, G_{23}=3.48 \mathrm{GPa}$ & $\varphi_{n}^{c}=\varphi_{t}^{c}=57.31 \mathrm{~Pa} \cdot \mathrm{m}$ \\
\hline
\end{tabular}

TABLE 2: Comparison of different calculation methods.

\begin{tabular}{|c|c|c|c|c|}
\hline Calculation method & Element number & Node number & $\begin{array}{c}\text { Strain energy release } \\
\text { rate } / \mathrm{J} / \mathrm{mm}^{2}\end{array}$ & Relative error/\% \\
\hline $\begin{array}{l}\text { Experimental values } \\
{[15]}\end{array}$ & - & - & $258 \pm 12$ & - \\
\hline WFEM & $\begin{array}{c}2 \text { wavelet plane plate } \\
\text { elements and } \\
1 \text { wavelet cohesive interface } \\
\text { element }\end{array}$ & 180 & 243.60 & 5.58 \\
\hline CFEM & $\begin{array}{l}1400 \text { PLANE183 and } 140 \\
\text { INTER202 }\end{array}$ & 2750 & 230.43 & 10.85 \\
\hline
\end{tabular}

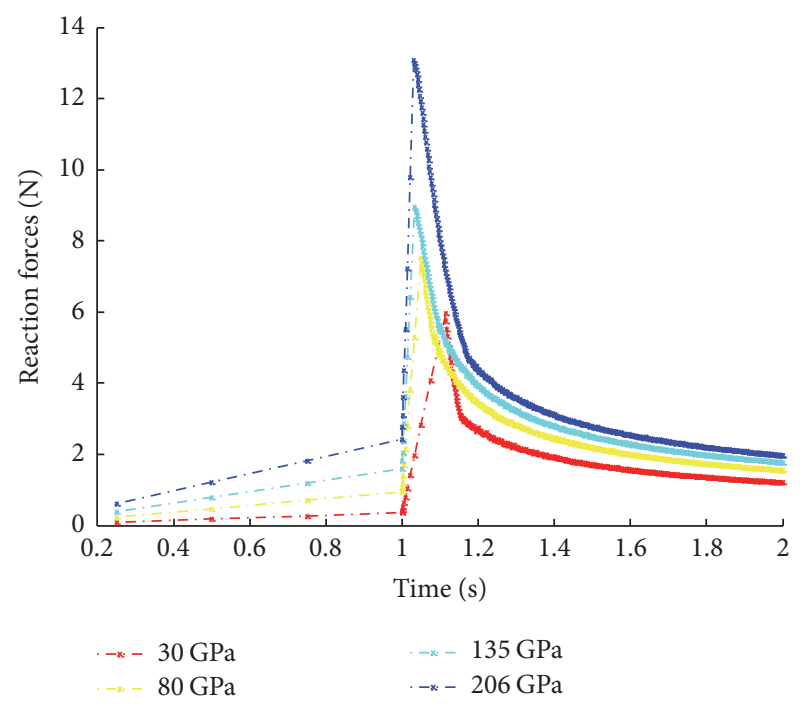

FIGURE 4: Reaction forces trends for different elastic modulus values.

different elastic modulus ratio, as presented in Figure 5. It can be concluded that the stress at the crack tip increases with

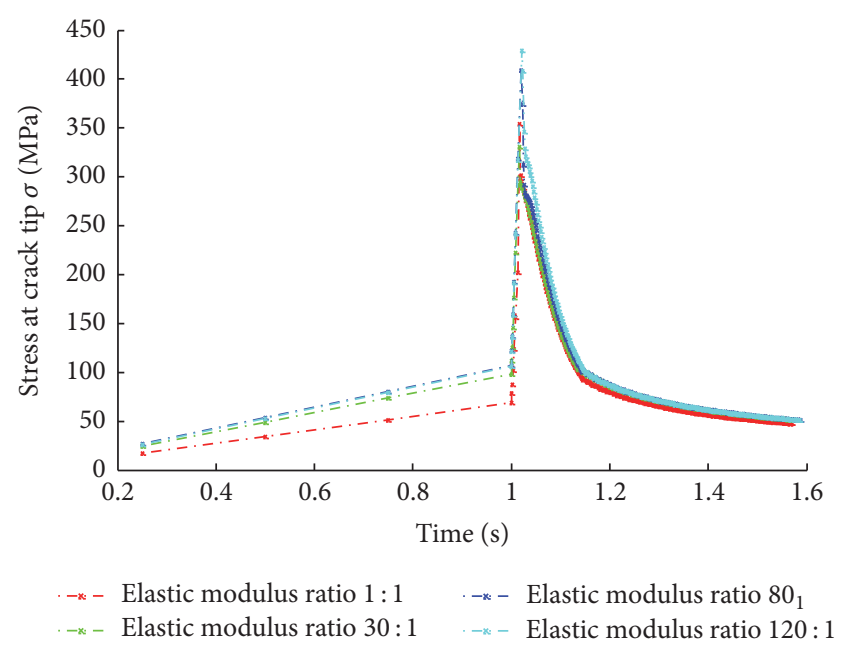

FIGURE 5: Stress at the crack tip under different elastic modulus ratio.

the increase of elastic modulus ratio; the higher the elastic modulus ratio is the faster the interface crack grows.

Figure 6 shows the effect of different initial crack length values on the interface crack growth. As can be observed 


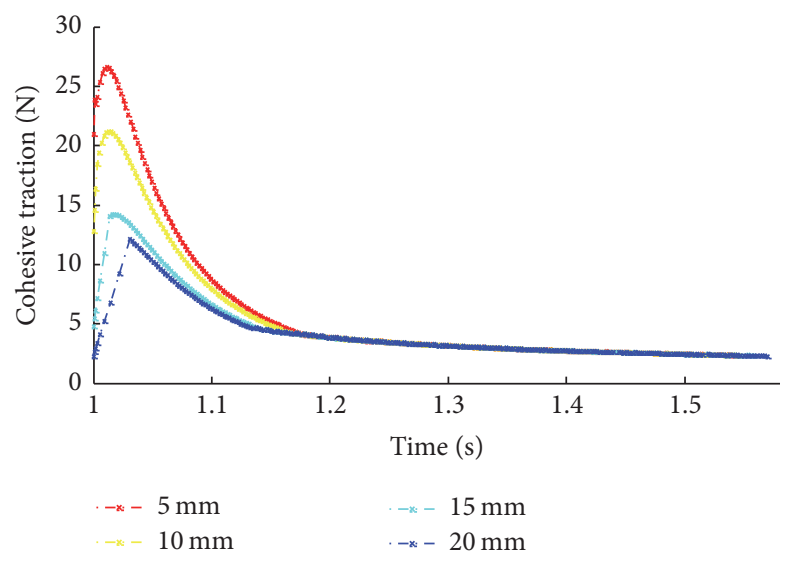

FIgURE 6: The variation law of cohesive traction and time for different initial crack length values.
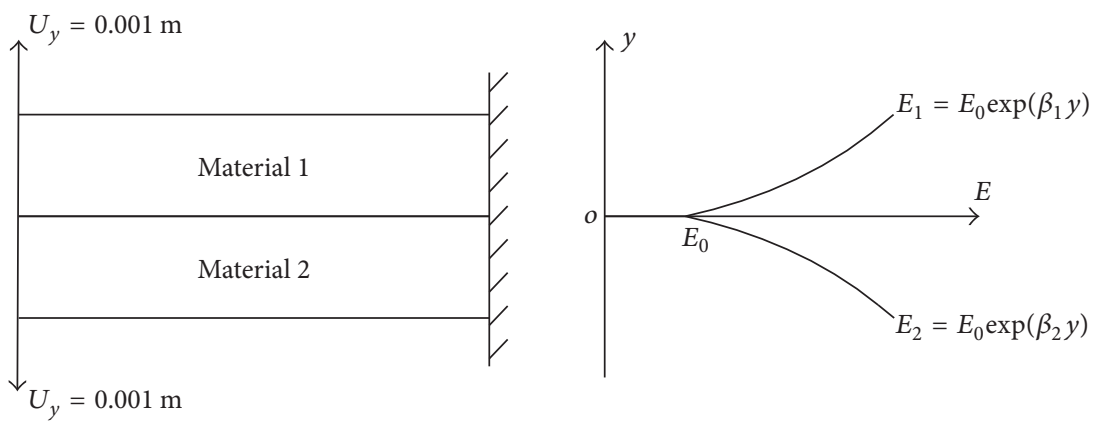

FIGURE 7: DCB interface crack growth model and its nonuniform material.

from Figure 6, the longer the initial crack length of DCB, the more pronounced the cohesive traction of DCB will be. This indicates that the longer the initial crack length is, the easier the interface crack grows.

\subsection{Interface Crack Growth Problem of Nonuniform Material} $D C B$. As presented in Figure 7, the geometrical dimensions and materials parameters of nonuniform material DCB are beam length $L=300 \mathrm{~mm}$, beam height $H=32 \mathrm{~mm}$, the initial crack length $a=30 \mathrm{~mm}$, elastic modulus $E_{0}=$ $2.0 \times 10^{11} \mathrm{~Pa}$, and Poisson ratio $\mu=0.3$. The corresponding interface parameters are maximum normal traction $T_{\max }=$ $95 \mathrm{MPa}$ and normal separation displacement $\delta_{n}=0.006 \mathrm{~mm}$.

Table 3 presents the interface stress at the crack tip of DCB under different nonuniform material parameters $\beta$. It can be observed by comparing the results of WFEM (484 DOFs) with CFEM (38400 DOFs) that WFEM and CFEM are in good agreement. Therefore, good computational accuracy and efficiency were further demonstrated. The relationship between the corresponding laws of interface stress as a function of time, under different parameters $\beta$, is demonstrated in Figure 8 . The curves show that the cohesive traction increases and the interface damage initiated when reaching the highest point. Then, it was successively decreased to an approximately constant value, which is consistent with interface damage

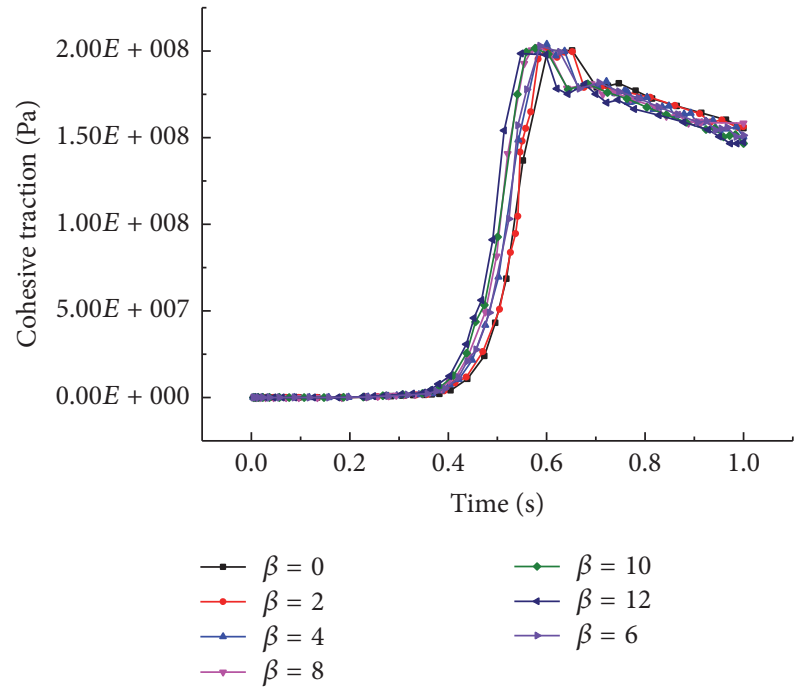

Figure 8: The variation law of cohesive traction and time.

process. Also, it was demonstrated that the interface damage initiated earlier, when the nonuniform parameter $\beta$ was at higher values; therefore, the uniform changing of material for DCB is beneficial for preventing crack growth. 
TABLE 3: Interface stress at the crack tip of nonuniform material DCB.

\begin{tabular}{lccc}
\hline \multirow{2}{*}{ Nonuniform material parameters $\beta$} & \multicolumn{3}{c}{ The interface stress at the crack tip/MPa } \\
& WFEM & CFEM & 148.79 \\
Relative error/\% \\
\hline 0 & 145.41 & 148.13 & 2.32 \\
4 & 145.26 & 128.49 & 1.98 \\
6 & 124.42 & 130.21 & 3.27 \\
8 & 126.23 & 135.19 & 19.15 \\
10 & 131.79 & 182.92 & 1.98 \\
12 & 179.36 & 188.05 & 3.33 \\
\hline
\end{tabular}

\section{Conclusions}

The new CZM based on the WFEM was constructed and the corresponding wavelet interface element stiffness matrix was obtained by (17). The relative error of the WFEM, when 3 elements and 360 nodes were employed with the experimental mean, was 5.58\%, while that of the CFEM, when 1540 elements and 5500 nodes were employed with the experimental mean, was $10.85 \%$, which indicated that the WFEM can obtain higher accuracy with less elements and nodes and it is suitable to solve the problem of interface crack growth.

The elastic modulus has a great impact on interface crack growth. The higher the elastic modulus of the material, the lower the required energy consumption of interface crack growth, and the faster the crack growth.

The study on the impact of the elastic modulus ratio of DCB on interface crack growth shows that, with the increase of the elastic modulus ratio of DCB, the stress concentration in interface crack tip increases, and interface crack is easier to extend. Therefore, interface crack growth can be postponed by adjusting the elastic modulus ratio. Also, the material parameters for DCB changes uniformly, which can prevent interface cracks from growing.

The study results of interface crack growth based on the initial length of interface crack show that cohesive traction decreases with the increase of initial length of interface crack; therefore, the interface cracks get easier growth.

$\begin{array}{ll}\text { Abbreviations } \\ i: & \text { Scale } \\ j: & \text { Order } \\ \phi_{m}^{j}: & \text { Scaling function } \\ \psi_{m}^{j}: & \text { Wavelet function } \\ \mathrm{BSWI}_{m j}: & j \text { scale } m \text { th order of BSWI } \\ \xi: & \text { Tangential coordinate, independent variable of } \\ & \text { wavelet scaling functions on the interval }[0,1] \\ T_{n}^{\text {max }}: & \text { Maximum normal cohesive traction, MPa } \\ \delta_{n}^{*}: & \text { Normal displacement jump at maximum } \\ & \text { normal cohesive traction, } \mathrm{m} \\ D_{n}: & \text { Interface damage parameter } \\ \delta_{n}^{c}: & \text { Normal displacement jump at the completion } \\ T_{n}: & \text { of debonding, m } \\ \delta_{n}: & \text { Interface normal cohesive traction }\end{array}$

$\Phi$ : Scaling functions vector

$\psi$ : Wavelet functions vector

$\otimes:$ Kronecker symbol

$\mathbf{u}^{e}$ : Interface element nodes displacement

$u$ : Continuous displacement field of upper and lower interface

$\mathbf{R}^{e}$ : Converted matrix

$\mathbf{K}$ : Interface element stiffness matrix

$\varphi_{n}^{c}$ : Normal critical fracture energy

$\varphi_{t}^{c}$ : Tangential critical fracture energy.

\section{Competing Interests}

The authors declare that they have no competing interests.

\section{Acknowledgments}

The work was financially supported by the National Natural Science Foundation of China (Grant no. 11502051), Major Project of Chinese Heilongjiang Province (Grant no. GA13A402), and Education Department Foundation of Chinese Heilongjiang Province (Grant no. 12541091).

\section{References}

[1] C. Li, Y. Tie, and Y.-P. Zheng, "Influence of material characters on bimaterial interfacial crack energy release rates and stress intensity factors," Journal of Mechanical Engineering, vol. 45, no. 3, pp. 104-108, 2009.

[2] T. Hu, The Numerical Simulation on Composite Materials Interface Crack Propagation Mechanism, Nanchang University, Nanchang, China, 2013.

[3] X.-F. Li and B.-L. Wang, "Anti-plane shear crack normal to and terminating at the interface of two bonded piezoelectric ceramics," International Journal of Solids and Structures, vol. 44, no. 11-12, pp. 3796-3810, 2007.

[4] R.-X. Bai and H.-R. Chen, "Numerical analysis of delamination growth for stiffened composite laminated plates," Applied Mathematics and Mechanics, vol. 17, no. 4, pp. 78-82, 2004.

[5] A. Miravete and M. A. Jiménez, "Application of the finite element method to prediction of onset of delamination growth," Applied Mechanics Reviews, vol. 55, no. 2, pp. 89-106, 2002.

[6] Y.-M. Cheng and M.-J. Chen, "A boundary element-free method for linear elasticity," Acta Mechanica Sinica, vol. 35, no. 2, pp. 181-186, 2003. 
[7] S.-C. Li, Y.-M. Cheng, and S.-C. Li, "Meshless manifold method for dynamic fracture mechanics," Acta Physica Sinica, vol. 55, no. 9, pp. 4760-4766, 2006.

[8] T. Belytschko, C. Parimi, N. Moës, N. Sukumar, and S. Usui, "Structured extended finite element methods for solids defined by implicit surfaces," International Journal for Numerical Methods in Engineering, vol. 56, no. 4, pp. 609-635, 2003.

[9] S. Mariani and U. Perego, "Extended finite element method for quasi-brittle fracture," International Journal for Numerical Methods in Engineering, vol. 58, no. 1, pp. 103-126, 2003.

[10] H. Zuo, Z.-B. Yang, X.-F. Chen, Y. Xie, and H. Miao, "Analysis of laminated composite plates using wavelet finite element method and higher-order plate theory," Composite Structures, vol. 131, pp. 248-258, 2015.

[11] J. W. Xiang, Y. X. Wang, and Z. S. Jiang, "Numerical simulation of plane crack using hermite cubic spline wavelet," CMES: Computer Modeling in Engineering \& Sciences, vol. 88, pp. 1-15, 2012.

[12] X. F. Chen, B. Li, Y. M. He, and Z. J. He, "Second generation wavelet finite element and rotor cracks quantitative identification method," Chinese Journal of Mechanical Engineering, vol. 23, no. 2, pp. 195-199, 2010.

[13] X.-F. Chen, Z.-J. He, B. Li, and J. Xiang, "An efficient wavelet finite element method in fault prognosis of incipient crack," Science in China Series E: Technological Sciences, vol. 49, no. 1, pp. 89-101, 2006.

[14] Y.-M. Wang and Q. Wu, "Construction of operator-orthogonal wavelet-based elements for adaptive analysis of thin plate bending problems," CMES: Computer Modeling in Engineering \& Sciences, vol. 93, no. 1, pp. 17-45, 2013.

[15] H.-F. Peng, G.-W. Meng, L.-M. Zhou et al., "Virtual crack closure technology based on wavelet finite element method," Journal of Jilin University (Engineering and Technology Edition), vol. 41, no. 5, pp. 1364-1368, 2011.

[16] Y. Zhong and J. Xiang, "Construction of wavelet-based elements for static and stability analysis of elastic problems," Acta Mechanica Solida Sinica, vol. 24, no. 4, pp. 355-364, 2011.

[17] Z. Yang, X. Chen, X. Zhang, and Z. He, "Free vibration and buckling analysis of plates using B-spline wavelet on the interval Mindlin element," Applied Mathematical Modelling, vol. 37, no. 5, pp. 3449-3466, 2013.

[18] Z. Yang, X. Chen, X. Li, Y. Jiang, H. Miao, and Z. He, "Wave motion analysis in arch structures via wavelet finite element method," Journal of Sound and Vibration, vol. 333, no. 2, pp. 446-469, 2014.

[19] Y.-M. Wang and Q. Wu, "Construction of operator-orthogonal wavelet-based elements for adaptive analysis of thin plate beanding problems," CMES: Computer Modeling in Engineering \& Sciences, vol. 93, pp. 17-45, 2013.

[20] P. D. Zavattieri, "Modeling of crack propagation in thin-walled structures using a cohesive model for shell elements," Journal of Applied Mechanics, vol. 73, no. 6, pp. 948-958, 2006.

[21] X. Chen, X. Deng, and M. A. Sutton, "Simulation of stable tearing crack growth events using the cohesive zone model approach," Engineering Fracture Mechanics, vol. 99, no. 2, pp. 223-238, 2013.

[22] B. E. Amstutz, M. A. Sutton, D. S. Dawicke, and J. C. Newman Jr., "An experimental study of CTOD for Mode I/II stable crack growth in thin 2024-T3 aluminum specimens," in Fracture Mechanics, ASTM STP 1256, pp. 256-271, American Society for Testing and Materials, 1995.
[23] T. Diehl, "On using a penalty-based cohesive-zone finite element approach, part I: elastic solution benchmarks," International Journal of Adhesion and Adhesives, vol. 28, no. 4-5, pp. 237-255, 2008.

[24] G.-F. Lu, Y. Liu, and C.-L. Zhang, "Analysis of strain energy release rate based on virtual crack closure technique," Acta Materiae Compositae Sinica, vol. 26, no. 2, pp. 210-216, 2009.

[25] D. Xie and S. B. Biggers Jr., "Progressive crack growth analysis using interface element based on the virtual crack closure technique," Finite Elements in Analysis and Design, vol. 42, no. 11, pp. 977-984, 2006.

[26] O.-Y. Chang, "On path-independent integrals and fracture criteria in nonlinear fracture dynamics," Applied Mathematics and Mechanics, vol. 3, no. 3, pp. 335-343, 1982.

[27] C. K. Chui and E. Quak, "Wavelets on a bounded interval," Numerical Methods of Approximation Theory, vol. 1, pp. 53-57, 1992.

[28] J. C. Goswami, A. K. Chan, and C. K. Chui, "On solving firstkind integral equations using wavelets on a bounded interval," IEEE Transactions on Antennas \& Propagation, vol. 43, no. 6, pp. 614-622, 1995.

[29] J. C. Goswami, A. K. Chan, and C. K. Chui, "Wavelet finite element theory and its engineering application," Science Press, vol. 43, pp. 614-622, 1995.

[30] I. Özdemir, W. A. M. Brekelmans, and M. G. D. Geers, "A thermo-mechanical cohesive zone model," Computational Mechanics, vol. 46, no. 5, pp. 735-745, 2010.

[31] A. Biel and U. Stigh, "Damage and plasticity in adhesive layer: an experimental study," International Journal of Fracture, vol. 165, no. 1, pp. 93-103, 2010.

[32] B. A. E. van Hal, R. H. J. Peerlings, M. G. D. Geers, and O. van der Sluis, "Cohesive zone modeling for structural integrity analysis of IC interconnects," Microelectronics Reliability, vol. 47, no. 8, pp. 1251-1261, 2007. 


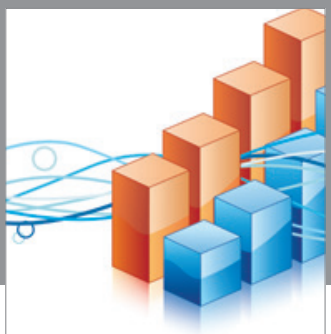

Advances in

Operations Research

vatem alat4

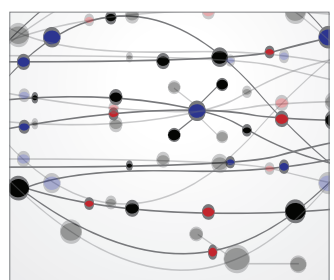

\section{The Scientific} World Journal
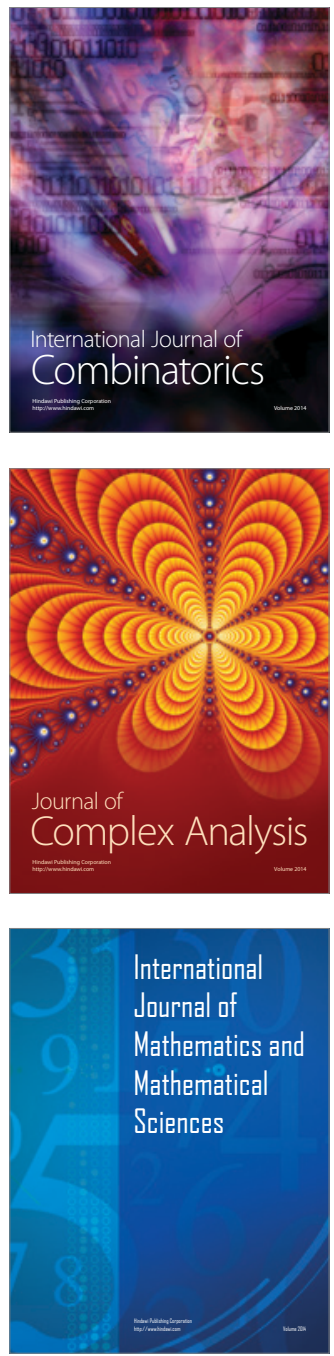
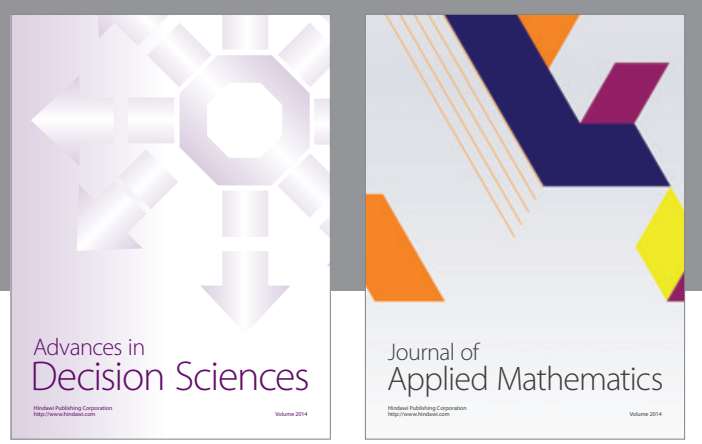

Algebra

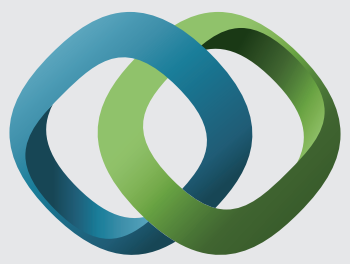

\section{Hindawi}

Submit your manuscripts at

https://www.hindawi.com
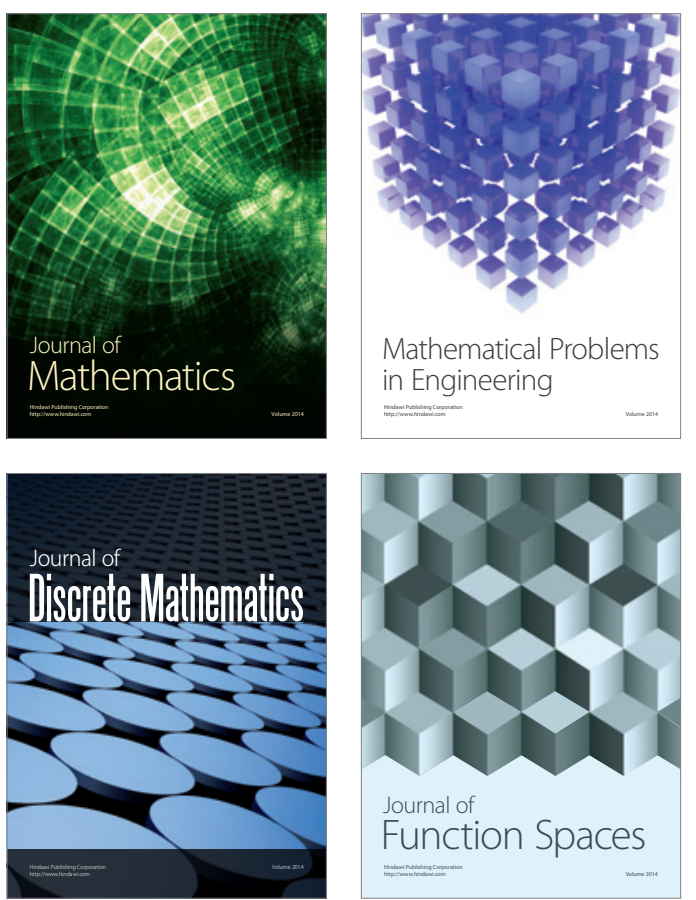

Mathematical Problems in Engineering
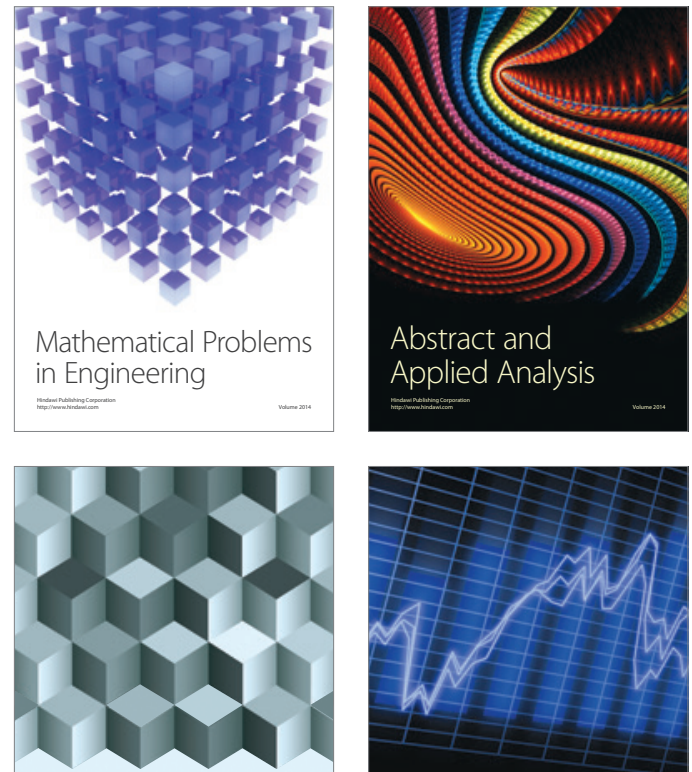

Journal of

Function Spaces

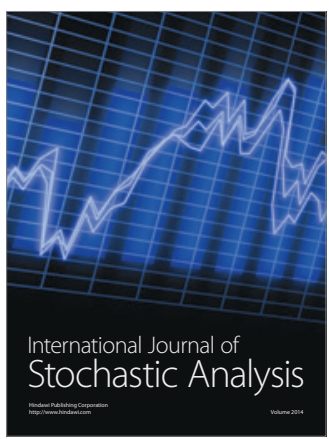

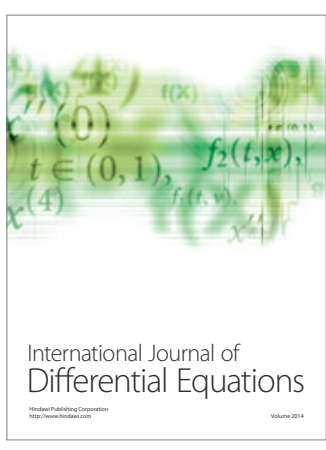
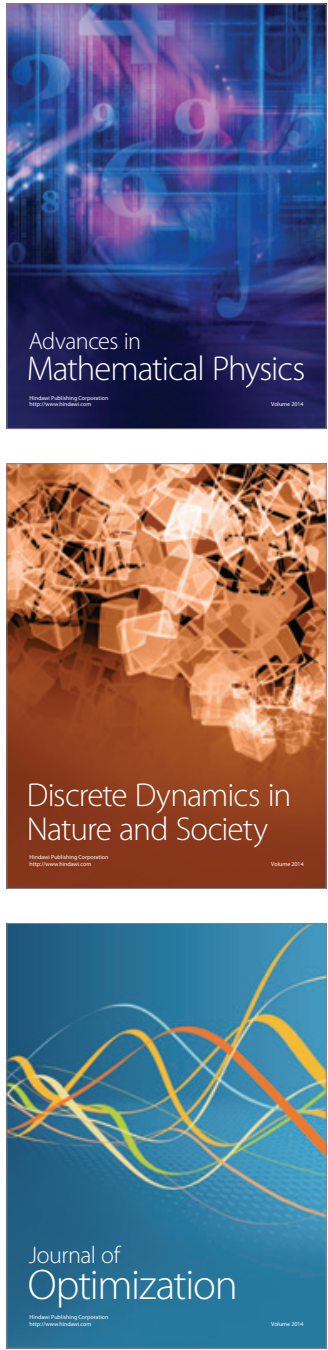